\title{
Perceptual Alternation Induced by Visual Transients
}

\author{
Ryota Kanai $^{1,4}$, Farshad Moradi ${ }^{2,4}$, Shinsuke Shimojo ${ }^{2,3}$ \& Frans A. J. Verstraten ${ }^{1}$ \\ ${ }^{1}$ Universiteit Utrecht, Helmholtz Research Institute, Psychonomics Division, \\ Heidelberglaan 2, NL-3584 CS Utrecht, The Netherlands \\ ${ }^{2}$ Computation and Neural Systems Program, California institute of Technology, \\ Pasadena, CA 91125 \\ ${ }^{3}$ NTT Communication Science laboratories (NTT Com. Sci. Lab.), Atsugi, Kanagawa, \\ 243 Japan. \\ ${ }^{4}$ These authors contributed equally to this work
}

Correspondence should be addressed to R.K.

Ryota Kanai

Universiteit Utrecht, Helmholtz Research Institute, Psychonomics Division, Heidelberglaan 2, NL-3584 CS Utrecht, The Netherlands

Email: r.kanai@fss.uu.nl

Tel. +31302533372

Fax +31302534511 


\begin{abstract}
When our visual system is confronted with ambiguous stimuli, the perceptual interpretation spontaneously alternates between the competing incompatible interpretations. The timing of such perceptual alternations is highly stochastic and the underlying neural mechanisms are poorly understood. Here, we show that perceptual alternations can be triggered by a transient stimulus presented nearby. The induction was tested for four types of bistable stimuli: structure-from-motion, binocular rivalry, Necker cube, and ambiguous apparent motion. While underlying mechanisms may vary among them, a transient flash induced time-locked perceptual alternations in all cases. The effect showed a clear dependency on the adaptation to the dominant percept prior to the presentation of a flash. These perceptual alternations show many similarities to perceptual disappearances induced by transient stimuli (Kanai \& Kamitani, 2003, Moradi \& Shimojo, 2004). Mechanisms linking these two transient induced phenomena are discussed.
\end{abstract}




\section{Introduction}

When we are presented with a stimulus that has multiple perceptual interpretations, we experience a sequence of spontaneous perceptual alternations between the possible interpretations. Such multistable stimuli have been used to dissociate perceptual from stimulus-driven mechanisms to study visual awareness. The timing of the alternations is highly variable, and the underlying mechanisms as well as the neural substrates are poorly understood. Understanding the mechanisms underlying the perceptual alternations is important not only for understanding bistable perception per se, but also for understanding the dynamics of normal vision (Blake \& Logothetis, 2002).

The perceptual alternations can be explained, to some extent, in terms of passive decays of perceptual signals (or adaptation). Although adaptation indeed increases the probability of alternations, it does not seem to directly cause them (Kohler \& Wallach, 1944; Hock, Schoner, \& Voss, 1997). That is, an additional factor seems necessary for an alternation to actually occur. Contributions of more active, top-down processes have been suggested to mediate alternations. Imaging studies have shown the involvement of parietal and frontal cortical areas (Lumer, Friston, \& Rees, 1998; Kleinschmidt et al., 1998). Also, bottom-up signals following small eye movements have been suggested to be responsible for perceptual alternations (Levelt, 1966, 1967, Murata et al. 2003; but see, Blake, Fox, \& McIntyre, 1971; Wade 1973). Since eye movements result in a transient signal due to new retinal input, the visual transient may contribute to or is even responsible for the induction of perceptual alternation. Surprisingly, to the best of our knowledge, this possibility has not been investigated systematically.

In this study, we show that a visual transient can often trigger a perceptual alternation. The induced alternations were time-locked to the transient, and therefore the timing is highly predictable. We show that presenting a flash behind a bistable figure results in a sudden transition of the current perception into the competing interpretation. This effect, termed Induced Perceptual Alternation (IPA), is demonstrated using a variety of 
ambiguous stimuli including structure-from-motion, binocular rivalry, Necker cube, and ambiguous apparent motion.

The IPA manifests characteristics similar to another transient-induced phenomenon where visual transients induce perceptual disappearance of objects (Kanai \& Kamitani, 2003; Moradi \& Shimojo, 2004). In both cases, transient stimuli cause a drastic change of the percept to a constant visual stimulus. We attempt to offer a schematic model that explains how visual transients produce a drastic perceptual change both in perceptual rivalry and disappearance.

\section{Experiment 1: Basic Effect}

In our first experiment, we show that perceptual reversals can be induced by transients in a variety of bistable stimuli; structure-from-motion, binocular rivalry, Necker cube, and bistable apparent motion.

\section{1. Methods}

\section{1. 1. Observers \& Apparatus}

Four naïve observers and one of the authors (RK) participated. All observers had normal or corrected-to-normal vision. The stimuli were generated on a Macintosh computer using Matlab PsychToolbox (Brainard, 1997; Pelli, 1997) and presented on a 22-inch CRT monitor (LaCie Blue Electron). The refresh rate of the display was $85 \mathrm{~Hz}$, and the resolution, $1280 \mathrm{X} 1024$. The viewing distance was $57 \mathrm{~cm}$.

\section{1. 2. Stimuli}

We displayed four types of bistable stimuli. These stimuli were Structure-From-Motion (SFM), Binocular Rivalry (BR), Necker Cube (NC), and bistable Apparent Motion (AM). The details of the stimulus parameters are described below. A fixation cross was placed in the geometric center of each stimulus. At random intervals every 2-4 s a transient signal (a brief white flash) was presented in the background. 
SFM (Figure 1a): The SFM consisted of 400 blue dots with a lifetime of 1 second presented against a black background. The shape of the structure was a cylinder rotating around the vertical axis centered at the fixation cross (red). The size of the cylinder was $3.9^{\circ}$ in height and $3.12^{\circ}$ in diameter. The rotation speed was 0.2 rps. For this stimulus, the transient was a flash that had a rectangular shape covering the whole stimulus area of SFM.

BR (Figure 1b): Two orthogonal square-wave gratings, red-black vs. green-black (45 deg and $135 \mathrm{deg}$ ) were presented in a circular area (diameter $7.8^{\circ}$ ). The gratings were presented dichoptically using red and green color filters. The gratings had a spatial frequency of $0.8 \mathrm{cpd}$ and the duty cycle was $20 \%$. To support binocular fusion, a white ring $\left(0.4^{\circ}\right.$ in width) was surrounding the stimulus. The combinations of color (red and green), eye (left and right) and orientation (45 deg and $135 \mathrm{deg}$ ) were randomly chosen for each trial. Transient flashes were presented by briefly changing the black part of the gratings (i.e. the background) into white for $47 \mathrm{~ms}$.

NC (Figure 1c): The skeletal drawing of a cube was presented in blue against a black background. The length of each edge was $1.95^{\circ}$ when viewed from a perpendicular angle. The flash was a solid white disk with a diameter of $7.81^{\circ}$ centering at the fixation.

AM (Figure 1d): AM was created by presenting a pair of blue disks alternately against a black background. The diameter of each disk was $0.98^{\circ}$. In one frame the disks were presented in the upper-right and lower-left quadrants, and in the other frame, in the upper-left and lower-right quadrants. These two frames were alternated every $200 \mathrm{~ms}$, and there was no blank interval between the frames. The four disk positions were $1.17^{\circ}$ away from the fixation point along both the horizontal and vertical axes. The flash was a white disk $\left(4.69^{\circ}\right.$ in diameter) centering at the fixation cross.

\section{1.3. Procedure}


Before starting the experiment, naïve observers were familiarized with bistable stimuli and perceptual reversals. It is known that it can be difficult for naïve observers to experience the first alternation (see, Girgus, Rock, \& Egatz, 1977; Rock \& Mitchener, 1992). In a trial, the observers viewed a bistable stimulus continuously for 30 seconds, and responded by a key press, when they experienced a perceptual reversal at any time during a trial: A flash (lasting for $47 \mathrm{~ms}$ ) was presented randomly between 2 and 4 seconds after the previous flash (uniform sampling). The flashes were always presented behind the ambiguous stimuli, without occluding any part of them.

As a control, we conducted the same experiment without the flashes to obtain the spontaneous reversal rates. Thus, there were 8 conditions (the flash and control conditions for each of four stimulus types). For each condition, the observer performed ten trials in a single session (the total viewing duration was 300 seconds). The order of the conditions was randomized for each observer.

\section{2. Results \& Discussion}

Figure 2 shows the frequency of alternation with respect to the flash onset. Alternations were observed often 500 1000 ms after the flash, indicating that sudden perceptual changes occurred just after the flash. The dashed lines show the frequency of alternation in the control experiment where the same stimuli were viewed but without the flash. Overall, the frequency of the alternation is significantly higher than the spontaneous alternation rates (t-test: SFM, $p<0.05$; BR, $p<0.01$; NC, $p<0.01$; AM, $p<0.05$ ).

The median of the time to alternation after the flash was presented was $706 \mathrm{~ms}, 612 \mathrm{~ms}$, $1024 \mathrm{~ms}$ and $824 \mathrm{~ms}$ for SFM, BR, NC, and AM, respectively. These values are larger than reaction times for simple detection tasks $(<500 \mathrm{~ms})$. This suggests that observers responded to the percept that occurred after the flash, and did not simply reacted to the sudden flash. Figure 3 shows the mean alternation rates for each stimulus type. In all stimulus types, the rates were higher in the flash condition compared with the control conditions (SFM, $p<0.01$; BR, $p<0.05$; NC, $p<0.01$; AM, $p<0.01$ ), 
The IPA seems more remarkable in the conditions with SFM and AM compared to BR and NC (Figure 2a). Why the flash was more effective at inducing alternations in these two types of stimuli is unclear. A difference between SFM/AM and BR/NC is that the former stimuli are dynamic, i.e. continuously moving, whereas the latter stimuli are constant. Thus, IPA may possibly have a stronger effect with dynamic stimuli.

\section{Experiment 2: Spatial Specificity}

In Experiment 1, we showed that a flash indeed induces alternations of the perceptual interpretation of ambiguous stimuli. However, the flash was always presented at the same location as the ambiguous stimuli. In the next experiment, we address the question as to whether the IPA is spatially limited to the location of the flash or caused by any transient stimuli.

We compared two conditions while presenting bistable stimuli in the periphery: 1) flash was presented at the same location as a bistable stimulus, and 2) flash was presented on the other side visual field (same eccentricity).

\section{1. Methods}

\section{1. 1. Observers \& Apparatus}

Five new naïve observers participated in the experiment with SFM and four naïve observers in the experiment using NC. Control data were obtained from three observers who participated in both experiments. Stimuli were presented on a CRT monitor with a resolution of $1152 \mathrm{X} \mathrm{864}$, and a refresh rate of $85 \mathrm{~Hz}$, and were viewed binocularly from $57 \mathrm{~cm}$.

\section{1. 2. Stimuli}

We chose to use two types of bistable stimuli, i.e. SFM and NC, as an example of dynamic and static ambiguous stimuli, respectively. We describe the details of the parameters used for these stimuli below. 
SFM: An imaginary cylinder consisted of 200 white dots (3.5 arcmin) against a black background. The cylinder subtended $4.4 \mathrm{deg}$ x $4.4 \mathrm{deg}$, and presented at an eccentricity of $2.93^{\circ}$ from the nearest edge to fixation on the left visual field (LVF). The cylinder rotated around a horizontal axis at $0.9 \mathrm{rps}$.

NC: A Necker cube (4.4 deg x $4.4 \mathrm{deg}$ ) was displayed in LVF. The edge nearest to the center of the screen was $2.93^{\circ}$ away to the left. The NC was presented on a gray background. Steady fixation while viewing a stationary object in the periphery results in a disappearance of the object due to adaptation (Troxler 1804), especially when transient stimuli are presented nearby (Kanai \& Kamitani 2003; Moradi \& Shimojo, 2004). To avoid retinal adaptation and perceptual disappearance during observation, we had observers track a small dot ( 7 arcmin, arcmin from the center) slowly rotating around a fixation cross (at $0.32 \mathrm{rps}$ ).

The bistable stimuli were presented on the left visual field. At a random timing between 3 and 5 seconds (uniform sampling), a white flash of the same size was briefly presented for $35 \mathrm{~ms}$. There were two conditions as to the position of the flash. In the ipsilateral condition, the flash was presented at the same location (i.e. LVF) as the bistable stimuli. In the contralateral condition (control), the flash was presented at the opposite location in the right visual field (RVF). Other factors - such as eccentricity, the duration of flash etc. - were similar.

\section{1. 3. Procedure}

Observers pressed a key to report the percept at the beginning of each session and when a percept switched to another. In a session, a bistable stimulus was shown continuously for 150 seconds. Three observers performed 4 sessions (2 ipsilateral sessions and 2 contralateral sessions). Two observers participated only in the ipsilateral condition. The order of experiments was counterbalanced within and between the observers.

\section{2. Results \& Discussion}


The frequency of perceptual alternations is plotted as a function of the time elapsed after the flash was presented (Figure 3). It can be seen from the histograms that in the ipsilateral condition, the alternations occurred following the flash. This resulted in a high frequency of reversals around 500 to $1000 \mathrm{~ms}$, both in the SFM (Figure 3a) and in the NC condition (Figure 3c). This was followed by a reduction of the frequency around $1.5 \mathrm{~s}$. In contrast, these peaks were not evident in the contralateral condition where the flash was presented in the opposite visual field (Figure 3b,d). The mean time of alternations with respect to the flash was significantly smaller in the ipsilateral condition (KruskalWallis ANOVA, $p<0.001$ in both SFM and NC). Also, the probability of reversals - as measured by the number of alternations per flash - was significantly larger in the ipsilateral condition than in the contralateral condition (SFM; 1.133 vs. $0.736, \chi^{2}=17.28$, $p<0.001$; NC; 0.925 vs. $\left.0.739, \chi^{2}=7.21, p<0.01\right)$.

The results indicate that the IPA retains a spatial specificity. That is, perceptual alternation is not triggered just by any transient event visible to the observers, but is limited to the areas around the flash. This spatial specificity is informative as to the level where the flash interacts with the representations for bistable stimuli. It shows that the interaction is presumably occurring at relatively early visual areas where visual inputs are retinotopically organized.

The fact that the flash presented on the opposite side does not induce perceptual alternation also suggests that the IPA is not caused by a secondary effect. For example, the perceptual alternations could be produced by a secondary effect of the peripheral flash like eye movements or eye blinks (but see Tse, Sheinberg \& Logothetis, 2002). If the flash were to induce eye movements or blinks, perceptual alternations would be expected to occur also in the contralateral condition. However, this was not the case. Thus, the IPA appears to be mediated via local interactions between a transient stimulus and the representations for bistable stimuli.

\section{Experiment 3: Location Specificity versus Perceptual Grouping}


Experiment 2 demonstrates that presenting a flash at the same location as the bistable stimulus induces perceptual alternation, whereas a flash in the opposite visual hemifield does not show the effect. In other words, for the IPA to occur, the flash has to be presented at the same location as the target.

Is the absence of IPA in the contralateral condition solely due to the large spatial separation? Here, we are interested in characterizing the nature of this spatial specificity. In particular, we attempt to address the question of whether the spatial specificity is defined in an object-based manner, or solely based on spatial separation.

We examined whether a flash presented on a distant, but perceptually grouped object can induce an alternation. We presented two SFM cylinders in the LVF, rotating around a vertical axis. In this configuration, both cylinders are perceptually grouped together and often perceived to rotate in the same direction (Eby, Loomis, \& Solomon, 1989; Gillam, 1972, Grossmann \& Dobbins, 2003). Observers were asked to report reversals for just one of the cylinders (target), while a flash was presented either at the target or non-target locations. If perceptual alternation operates in an object-based fashion, then presenting a flash at either location should induce alternations.

In these tasks, attention is conceivably directed only to the target. This attentional bias may disrupt the perceptual grouping of the two cylinders. Therefore, these tasks could possibly undermine the grouping effect. Thus, using the same configuration, we also performed an experiment where observers were asked to report whether both cylinders are moving in the same direction, or in the opposite directions. This task requires the observers to attend both cylinders.

\section{1. Methods}

Eleven naïve observers participated in the experiment. Two SFM stimuli (cylinders) were presented $\pm 0.6 \mathrm{deg}$ apart from the horizontal meridian (Figure 4a-d). Thus, the distance between the cylinders (edge-to-edge) was $1.2 \mathrm{deg}$. At a random timing between 
3 and 5 seconds (uniform sampling), a white flash of the same size was presented at the same location as one of the stimuli. The apparatus and stimuli parameters were identical to experiment 2 ,

Five conditions were tested in separate blocks. In conditions 1-3, the two cylinders were rotating around the vertical axis, and observers were asked to report the reversals of the top (condition $1, \mathrm{n}=10$ ), or bottom cylinder (condition $2, \mathrm{n}=11$ ), or if both cylinders are rotating in the same direction (condition $3, \mathrm{n}=10$ ). In conditions $4-5$, the two cylinders had orthogonal axes. Unlike coaxial configuration, spontaneous perceptual alternations are not linked for orthogonal SFM cylinders. Observers were asked to report the reversals of the top (condition $4, n=7$ ), or bottom cylinder (condition $5, n=9$ ). The target cylinder was always rotating around the vertical axis.

The SFM stimuli were presented continuously in each block for $200 \mathrm{~s}$. The order of the experiments was randomized for each observer. In all the conditions, the flash occurred at the target location or the non-target location randomly.

\section{2. Results and Discussion}

The results for each stimulus condition are shown in Figure 4a-d, and the number of induced reversals (i.e., reversals occurring within $0.5-1 \mathrm{~s}$ after the flash) for each condition is summarized in Table 1. As in the previous experiments, perceptual alternations were successfully induced when the flash was presented at the location of the target (Figure 4e-h).

Our interest here is whether an alternation is induced when the flash is presented on a spatially separate, but perceptually grouped stimulus. The results were ambiguous as to this question. In fact, we found an asymmetrical effect between the upper and lower visual fields for coaxial SFM. When the target was the cylinder in the upper visual quadrant (condition 1, Figure 4e), there was an effect of perceptual grouping. That is, the flash on the lower cylinder produced a time-locked perceptual alternation of the top cylinder. Indeed, the IPA occurred about equally frequently independent of the flash 
position (condition 1: same vs. different, $p=0.92$ ). On the other hand, when the target was the lower cylinder (condition 2, Figure 4i), the flash on the upper cylinder did not produce the IPA of the lower cylinder (condition 2, same vs. other: $p<0.001$, Chi-square test). This shows that the IPA is spatially very specific to the target position, when the target is in the lower visual field.

Why is there such an asymmetry between the upper and lower visual quadrants? Perceptual grouping between the top and bottom cylinder seems to be an important factor, because IPA was confined to the target location for orthogonal configuration of SFM stimuli, regardless of the target being in the upper or lower visual quadrant (Figure 4k,1). Another possible factor is attentional resolution, which is also known to manifest an upper-lower asymmetry (He, Cavanagh, \& Intriligator, 1996). Since attentional resolution is higher in the lower visual field, attention to the bottom cylinder may more effectively individuate the target stimulus from the other cylinder. If so, the perceptual grouping of the two cylinders would be weaker when the lower cylinder is attended.

When the observers were required to attend to both cylinders (condition 3), the flash disrupted the synchronous rotations of the two cylinders (Figure 5a), which often took 0$1 \mathrm{~s}$ to recover (Figure 5b). We did not find a noticeable difference in the effect depending on the flash position (top or bottom) as in the other conditions where the observers were monitoring the rotation direction of either cylinder. Three observers reported that the flash induced anti-rotation quite frequently. This implies that for these observers, the flash had often a local effect. However, other observers experienced the anti-rotation rarely, as if the rotations of both cylinders are always linked. In other words, the flash either induced an alternation of both cylinders simultaneously or did not affect either of them. These observations suggest that a flash has an effect on a slightly larger scale. That is, the effect is not just confined to the location the flash, but spreads along a perceptually grouped object.

Taken together, the IPA manifests both a spatial specificity and an effect of perceptual grouping depending on the target position. Currently, we cannot specify what are other 
factors that determine on which principle the IPA operates. However, as we discussed above, one of the likely candidates is attention. When a local aspect of a stimulus is attended, it will be relatively isolated from the rest. In such a situation, the IPA is limited within this area, i.e. a flash outside the attended part does not induce an alternation. On the other hand, when attention is directed to an object as a whole (two cylinders as one grouped object), a transient stimulus presented within this object seems to have a global impact on the entire object including the portions that are not directly stimulated by the transient.

\section{Experiment 4: Effects of Adaptation}

In the previous experiments, we presented a flash repeatedly and showed that the flash is responsible for the increase in the frequency of perceptual reversals. In bistable perception, adaptation to the dominant percept is widely considered as one of the key factors causing perceptual alternations (Köhler \& Wallach, 1944; Cornwell, 1976; Babich \& Standing, 1981; Merk \& Schnakenberg, 2002; Blake, Sobel \& Gilroy, 2003). How is the effect of a flash related to the adaptation process in bistable percepts? The purpose of the next experiment is to examine the possible interactions between a transient stimulus and adaptation. Prior to a flash, we presented bistable stimuli for a variable duration of time, and examined the dependency of IPA on the adaptation duration.

If the flash directly causes an alternation independent of sensory adaptation to a dominant percept, the alternation frequency will not depend on the adaptation duration. Alternatively, if adaptation does have an effect on the IPA, the frequency of IPA will depend on the adaptation duration.

\section{1. Methods}

\section{5 .1. 1. Observers \& Apparatus}

Six observers including the author (RK) participated in this experiment. One observer was excluded from the analysis because he had difficulty in perceiving perceptual alternations in SFM, even when he continuously viewed the stimulus for several minutes. The stimuli were presented on a 22-inch CRT monitor (LaCie Blue Electron). The 
refresh rate of the display was $60 \mathrm{~Hz}$, and the resolution $1280 \mathrm{X} 1024$. Viewing distance was $57 \mathrm{~cm}$.

\subsection{Stimuli}

In this experiment, we used SFM and NC. One trial consisted of three phases; adaptation phase, flash phase, and post-flash phase (Figure 6a). In all phases, a bistable stimulus was continuously shown on a black background. The duration of adaptation phase was varied between $250 \mathrm{~ms}, 500 \mathrm{~ms}, 1000 \mathrm{~ms}, 2000 \mathrm{~ms}$, and $3000 \mathrm{~ms}$. In the flash phase, the background of stimulus turned into white for $82 \mathrm{~ms}$. Subsequently, the same stimulus was observed for another $500 \mathrm{~ms}$ so that observers could make a judgment as to whether there was a perceptual reversal or not.

The exact parameters used in this experiment are described below. The center of the stimuli was located $5.9^{\circ}$ left to the fixation cross.

SFM: The cylinder of the SFM-stimulus had a radius of $1.9^{\circ}$ of visual angle and a height of $4.5^{\circ}$. It consisted of 800 blue dots with a lifetime of $583 \mathrm{~ms}$ (35 frames) and rotated at a speed of $0.5 \mathrm{rps}$.

NC: The Necker cube was drawn in blue subtending approximately $2.5^{\circ}$ of visual angle when viewed from the perpendicular angle.

\section{1. 3. Procedure}

At the end of each trial, observers reported whether perceptual reversals occurred just after the flash. When spontaneous reversals occurred before the flash, the trial was repeated. Observers were instructed to attend to the initial percept once the trial was started. This manipulation of attention is known to keep spontaneous alternations lower (Pelton \& Solley, 1968), thus preventing frequent early alternations before the flash. Observers performed 20 trials for each condition.

\section{2. Results \& Discussion}


The results for the SFM and NC stimuli are displayed in Figure 6b,c. The proportion of trials for which the flash triggered a perceptual alternation is shown as a function of adaptation duration. The results show that the flash was more effective at inducing an alternation when one interpretation had been perceived for a longer time, which results in deeper adaptation. The alternations increased monotonically as the adaptation duration increased (Spearman's rank order correlation: $\mathrm{R}=0.79, p<0.001$ for $\mathrm{NC}, \mathrm{R}=0.65$, $p<0.001$ for SFM). The results indicate that some adaptation is required for an alternation to occur and a flash by itself is not necessarily sufficient.

\section{General Discussion}

We have shown that perceptual reversals during the observation of ambiguous stimuli can be induced by a transient stimulus. This Induced Perceptual Alternation (IPA) provides us with a means to investigate the dynamics underlying the perceptual alternation.

Our experiments revealed the basic characteristics of the IPA. First, the reversals are time-locked to the flash. Second, the effect is largely confined to the location of the flash. That is, the flash needs to be presented near the target stimulus. Third, adaptation to the dominant perceptual interpretation is necessary, and the strength of the effect depends on the depth of adaptation.

These characteristics are shared by another transient-induced phenomenon, where a transient stimulus triggers a fading of an object presented in the periphery (Kanai \& Kamitani, 2003; Moradi \& Shimojo, 2004; see also, Wilke, Logothetis \& Leopold, 2003; Breitmeyer \& Rudd, 1981). The fading effect shows the time-locked characteristics. It is confined to a limited spatial region near the transient stimulus. Also, pre-adaptation, although brief, is necessary for a fading to be reliably triggered. In addition to the similarities in these basic characteristics, what is common between the perceptual alternations and disappearances, is that conscious perception for a physically constant stimulus is drastically changed by transient stimuli. In fact, results of other studies also suggest that there is a common mechanism underlying both perceptual disappearance and 
bistable percepts (Bonneh, Cooperman, \& Sagi, 2000; Carter \& Pettigrew, 2003). Given these similarities between perceptual alternations and fading, we try to understand the function of a visual transient both in the perceptual fading and the IPA in a unified scheme. Here we address this issue in two parts: first, what kind of dynamics can result in such a behavior, and second, what is the possible underlying neural mechanism for such dynamics.

\section{Neural Model and Simulation}

We propose a schematic model of the effects of transient input that incorporates both the alternation and fading effects. Perceptual bi-stability is often considered analogous to the alternation between states or attractors in bi-or multi-stable neural networks or dynamical systems (Poston \& Stewart, 1978; Kawamoto \& Anderson, 1985). Stability can be analyzed in such models by constructing a so-called Energy function (or Lyapunov function) such that in the absence of noise the energy always decreases until the system reaches a fixed state or a stable attractor. The stable states of such models are determined by local minima of the energy function. In Figure 7, two mutually exclusive percepts for a bistable stimulus are illustrated as two locally stable minima in the energy function (left and right).

Experimental evidence suggests that prior adaptation can increase the reversal rate from the adapted percept to the unadapted percept (Long, Toppino \& Mondin, 1992). This finding is consistent with a scheme in which adaptation changes the energy landscape of the system by increasing the energy of the adapted state (Figure 7c). Conceivably, a transient stimulus at the same location should induce a reversal either by changing the state of the network, or by modifying the energy landscape.

It has been suggested that a salient transient signal triggers a rapid change in the gain of the input (Moradi \& Shimojo, 2004). This type of response is necessary for efficient coding of information in a changing environment. In the absence of the transient signal, the gain gradually returns to the level before the flash. However, the change in the input 
gain following a flash results in smoothing of the energy landscape (Figure 7b). Consequently, our model predicts that the spontaneous reversal rate increases following the flash. However, if a flash follows sufficient adaptation, the combined effect of adaptation and reduction of the gain makes the previous local minima unstable and as a result, induces a perceptual alternation ${ }^{1}$ (Figure $7 d$ ).

Figure 8 demonstrates a minimal implementation of the fore mentioned schematic account. We modeled the bi-stability in our network using opponent neurons (that selectively respond to opposite directions) with symmetric inhibitory connections (Figure 8a). When there is no noise in the network, the state always converges to one of the two stable minima (percepts) and the outcome is determined by the initial condition. In the presence of stochasticity, however, the system occasionally alternates between the two percepts. The rate for switching from one state to another depends on the difference between their energies and noise level, as well as the height of the energy separating the minima.

The output of each Ising neuron at each time-step is modeled as follows (Hinton \& Sejnowski, 1986):

$$
\text { Output }= \begin{cases}1 & \text { With probability } \frac{1}{1+e^{-2(a x-b)}} \\ -1 & \text { Otherwise }\end{cases}
$$

where $x$ is the total input given $w_{e}=-w_{i}=1, a=2.5$ is the slope, and $b$ reflects the change in threshold following adaptation:

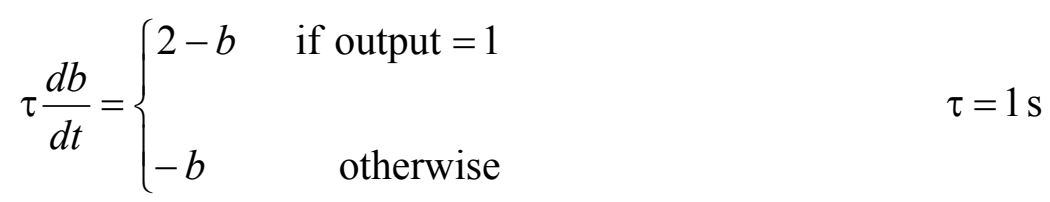

\footnotetext{
${ }^{1}$ The observer who did not perceive any perceptual alternation in the SFM condition in Experiment 4 reported that flash makes the SFM flat temporarily, and the cylinder shape restores after some duration. This observation is consistent with our interpretation that a flash makes the energy landscape smooth. In this observer, energy minimum for one interpretation was predominant, and the smoothing effect could only make the minimum briefly unstable, and was not sufficient to induce an alternation.
} 
Figures $8 \mathrm{~b}$ compares the distribution of intervals between perceptual reversals in Experiment 3 (SFM-contra, actual data) and simulation data. This rudimentary model seems to at least qualitatively capture the stochastic nature of spontaneous perceptual alternations.

The effect of transient was modeled by a transient decrease in the slope as follows:

$$
a(t)=a-k\left(e^{-\frac{t-t_{0}}{\tau_{2}}}-e^{-\frac{t-t_{0}}{\tau_{1}}}\right), \quad t>t_{0}
$$

where $\tau_{1}=150 \mathrm{~ms}, \tau_{2}=300 \mathrm{~ms}, k=3$, and $t_{0}$ reflects the onset of the transient plus $150 \mathrm{~ms}$ visual processing delay. Figure 8c demonstrates that such reduction in the slope can induce reversals comparable to the empirical data in Figure 4a (reaction-time is not included in the simulation). We also verified that if the transient slope change occurs before enough adaptation, it fails to induce any alternation (Figure 8d).

Thus, our model can explain the time-locked nature, and the requirement of adaptation in IPA. Moreover, it covers both the perceptual alternation and disappearance phenomena (Moradi \& Shimojo, 2004) induced by visual transients.

\section{Underlying Neural Mechanism}

Experiment 4 suggests that IPA results from a combination of two components, i.e. adaptation to a dominant percept and the effect of a transient. What are the neural foundations underlying these components? The adaptation process is specific for the stimulus and is likely to occur in early visual areas where visual inputs are still retinotopically organized. For example, it is known that adaptation to a Necker cube is specific to the location at which the stimulus is presented. Alternation rates of Necker cube usually increase during continuous viewing. However, the accelerated alternation rate due to adaptation restores to the original level, when the cube is moved to elsewhere in the visual field (Babich, \& Standing, 1981). Recently, Blake, Sobel and Gilroy (2003) have shown that bistable stimuli (BR and SFM) that are moving continuously in the 
visual field manifest slower alternation rates. This indicates also the involvement of location specific (retinotopic) adaptation.

On the other hand, the neural circuitry responsible for the effect of a flash is somewhat elusive. The increase in gain and the smoothing of the energy landscape in our proposed model can be achieved biophysically via non-specific shunting inhibition (Torre \& Poggio, 1978). Whether this is mediated through local interactions or attentional mechanisms via top-down feedback remains unclear. Nonetheless, we are tempted to attribute this effect of visual transients to a top-down mechanism mediated by the parietal attention system. The involvement of the parietal system in both the perceptual disappearance and perceptual alternation has been suggested before. Bonneh et al (2001) compared motion-induced blindness to the extinction of salient stimuli experienced by patients with parietal lesions. Parietal patients often fail to perceive a salient object presented contralateral to the damage cortical hemisphere (Driver \& Vuilleumier, 2001; Rees et al. 2000). Moreover, their perceptual disappearance is facilitated (Mennemeier et al., 1994; Wolpert, Goodbody, \& Husain, 1998).

Our preliminary observations showed that the spatial specificity of IPA is not strictly confined to the location of the target stimulus. When a flash was presented just above the mid-line target in a configuration similar to Experiment 2, the IPA was still observed. This mild form of spatial specificity is also observed in perceptual disappearance induced by transient stimuli (Kanai \& Kamitani, 2003; Wilke, Logothetis \& Leopold, 2003; Moradi \& Shimojo, 2004). This extended spatial specificity is in contrast with sensory adaptation that typically requires strict stimulus overlap.

The finding that a flash sometimes influences the percept in an object-based manner (see Experiment 3), also seems to suggest the involvement of a slightly higher mechanism rather than non-selective effects in the lower sensory areas. In Experiment 3, a flash presented on a coaxial SFM cylinder sometimes induced an alternation in other cylinder, which was presumably perceptually grouped. This implies that the effect of a flash transferred to a distant, but grouped object. Previously, a similar effect has been reported 
in perceptual disappearance (Kanai \& Kamitani, 2003). When a long bar is presented in a periphery, presenting a flash at one end of the bar, was sometimes sufficient to induce a fading of the entire bar. This also suggests an object-based effect of visual transients.

These comparisons suggest that there is a connection between perceptual fading and frontoparietal functions. Moreover, recent evidence indicates the involvement of topdown feedback in the perception of bistable stimuli. For example, the right frontoparietal cortex is involved in the disambiguation of bistable stimuli such as the Necker cube (Bisiach et al., 1999; Sengpiel, 2000; Inui et al., 2000). Also, frontoparietal areas associated with selective visual attention are considered to be involved in initiating perceptual alternation (Lumer, Rees, \& Friston 1998; Kleinschmidt, Büchel, Zeki, Frackowiak, 1998; Sterzer, Russ, Preibisch, \& Kleinschmidt, 2002; Leopold \& Logothetis 1999; Miller et al, 2000; Pettigrew, 2001). So the current evidence shows that frontoparietal areas play a critical role both in perceptual fading and alternation. This makes it tempting to suggest that these alternation-related areas may be the source of the inhibition by a flash, and causing IPA.

\section{Concluding remark}

In the field of the perception of bistable stimuli, there has been an extensive debate as to the mechanisms underlying perceptual alternations. The first explanation involves adaptation of the currently dominant stimulus interpretation (Kohler, 1940; Long and Toppino, 1994; Blake, Sobel, \& Gilroy, 2003). Proponents of the second explanation assume that attention-related processes actively trigger perceptual alternations (Lumer, Friston, \& Rees, 1998; Kleinschmidt et al., 1998; Leopold \& Logothetis, 1999). However, these explanations are not necessarily mutually exclusive and bistable perception seems to be mediated at a multitude of processing levels in the visual system (Blake \& Logothetis, 2002). Our model combines adaptation at lower sensory level and transient gain change via feedback. Finally, it is the first model that provides an account for both perceptual alternations and disappearances in a single scheme. 


\section{Acknowledgement}

We thank our observers for participating in the experiments Masataka Watanabe, DawAn Wu, Melanie Wilke, Chris Paffen, and Constanze Hofstötter for helpful discussion, Mark Changizi and Maarten van der Smagt for comments on a previous version of the manuscript. RK is funded by a grant from the Helmholtz Institute and FV by a grant of the Netherlands Organization for Scientific Research (NWO-Pionier). FM is supported by a fellowship from Caltech.

\section{References}

Babich S, Standing L, 1981 "Satiation effects with reversible figures" Perception and Motor Skills 52 203210

Bisiach E, Ricci R, Lai E, De Tanti A, Inzaghi M G,1999 "Unilateral neglect and disambiguation of the Necker cube" Brain 122 131-140

Blake R, Fox R, McIntyre C, 1971 "Stochastic properties of stabilized image binocular rivalry alternations" Journal of Experimental Psychology 88 327-332

Blake R, Logothetis NK, 2002 "Visual competition" Nature Reviews Neuroscience 3 1-11

Blake R, Sobel KV, Gilroy L A, 2003 "Visual motion retards alternations between conflicting perceptual interpretations" Neuron 39 869-78

Bonneh Y, Cooperman A, Sagi D, 2001 "Motion induced blindness in normal observers" Nature 411 798801

Brainard D H, 1997 “The Psychophysics Toolbox” Spatial Vision 10 433-436

Breitmeyer B, Rudd M E, 1981 “A single-transient masking paradigm” Perception and Psychophysics 30 604-606

Cornwell H G, 1976 "Necker cube reversal: Sensory or psychological satiation?" Perceptual and Motor Skills 43 3-10

Carter O L, Pettigrew J D, 2003 “A common oscillator for perceptual rivalries?” Perception 32 295-30

Driver J, Vuilleumier P, 2001 "Perceptual awareness and its loss in unilateral neglect and extinction" Cognition 79 39-88

Eby D W, Loomis J M, Solomon E M, 1989 "Perceptual linkage of multiple objects rotating in depth" Perception 18 427-444

Gillam B, 1972 "Perceived common rotary motion of ambiguous stimuli as a criterion of perceptual grouping" Perception and Psychophysics 11 99-101 
Girgus J J, Rock I, Egatz R, 1977 "The effect of knowledge of reversibility on the reversibility of ambiguous figures" Perception and Psychophysics 22 550-556

Grossmann J K, Dobbins A C, 2003 "Differential ambiguity reduces grouping of metastable objects" Vision Research 43 359-369

He S, Cavanagh P, Intriligator J, 1996 "Attentional resolution and the locus of visual awareness" Nature 383 334-337

Hinton G E, Sejnowski T J, 1986 "Learning and relearning in Boltzmann machines" in Parallel Distributed Processing: Explorations in the Microstructure of Cognition. Volume 1: Foundations Eds. D E Rumelhart, J L McClelland (Cambridge, MA: MIT Press)

Hock H S, Schoner G, Voss A, 1997 "The influence of adaptation and stochastic fluctuations on spontaneous perceptual changes for bistable stimuli" Perception and Psychophysics 59 509-522

Inui T, Tanaka S, Okada T, Nishizawa S, Katayama M, Konishi J, 2000 "Neural substrates for depth perception of the Necker cube; a functional magnetic resonance imaging study in human subjects" Neuroscience Letters 282 145-148

Kanai R, Kamitani Y, 2003 “Time-Locked Perceptual Fading Induced by Visual Transients" Journal of Cognitive Neuroscience 15 664-672.

Kawamoto A H, Anderson J A, 1985 "A neural network model of multistable perception" Acta Psychologica (Amsterdam) 59 35-65

Kleinschmidt A, Büchel C, Zeki S, Frackowiak S J, 1998 "Human brain activity during spontaneously reversing perception of ambiguous figures" Proceedings of the Royal Society of London B: Biological Sciences 265 2427-2433

Köhler W, 1940 Dynamics in psychology (New York: Liveright)

Köhler W, Wallach H, 1944 "Figural after-effects: An investigation of visual processes" Proceedings of the American Philosophical Society 88 269-357

Levelt W J M, 1966 “The alternation process in binocular rivalry” British Journal of Psychology 57 (3 and 4) $225-238$

Levelt W J M, 1967 "Note on the distribution of dominance times in binocular rivalry" British Journal of Psychology 58 (1and 2) 143-145

Long G M, Toppino T C, Mondin G W, 1992 "Prime time: fatigue and set effects in the perception of reversible figures" Perception and Psychophysics 52 609-616

Lumer E D, Friston K J, Rees G, 1998 "Neural correlates of perceptual rivalry in the human brain” Science 280 1930-1934

Leopold D A, Logothetis N K, 1999 "Multistable phenomena: changing views in perception" Trends in Cognitive Sciences 3 254-264

Mennemeier M S, Chatterjee A, Watson R T, Wertman E, Carter L P, Heilman K M, 1994 “Contributions of the parietal and frontal lobes to sustained attention and habituation" Neuropsychologia 6 703-716 
Miller S M, Liu G B, Ngo T T, Hooper G, Riek S, Carson R G, Pettigrew J D, 2000 "Interhemispheric switching mediates perceptual rivalry" Current Biology 10 383-392

Merk I, Schnakenberg J, 2002 “A stochastic model of multistable visual perception” Biological Cybernetics $86111-116$

Moradi F, Shimojo S, 2004 "Suppressive effect of sustained low-contrast adaptation followed by transient high-contrast on peripheral target detection" Vision Research 44 449-460

Murata T, Matsui N, Miyauchi S, Kakita Y, Yanagida T. 2003 "Discrete stochastic process underlying perceptual rivalry" Neuroreport 14 1347-1352

Pelli D G, 1997 "The VideoToolbox software for visual psychophysics: Transforming numbers into movies" Spatial Vision 10 437-442

Pelton L H Solley C M, 1968 "Acceleration of reversals of a Neckere cube" American. Journal of Psychology 81 585-588

Pettigrew J D, 2001 "Searching for the switch: Neural bases for perceptual rivalry alternations" Brain and Mind 2 85-118

Poston T, Stewart I, 1978 "Nonlinear modeling of multistable perception" Behavioral Sciences 23 318-334

Rees G, Wojciulik E, Clarke K, Husain M, Frith C, Driver J, 2000 “Unconscious activation of visual cortex in the damaged right hemisphere of a parietal patient with extinction" Brain 123 1624-1633

Rock I, Mitchener K, 1992 "Further evidence of failure of reversal of ambiguous figures by uninformed subjects" Perception 21 39-45

Sengpiel F, 2000 “An alternative view of perceptual rivalry" Current Biology 10 R482-485

Sterzer P, Russ M O, Preibisch C, Kleinschmidt A, 2002 "Neural correlates of spontaneous direction reversals in ambiguous apparent visual motion" Neuroimage 15 908-916

Torre V, Poggio T, 1978 "Synaptic mechanism possibly underlying directional selectivity to motion" Proceedings of the Royal Society of London B: Biological Sciences 202 409-416

Tse P U, Sheinberg D L, Logothetis N K 2002 "Fixational eye movements are not affected by abrupt onsets that capture attention" Vision Research 42 1663-1669

Wade N J, 1973 "Binocular rivalry and binocular fusion of afterimages" Vision Research 13 999-1000

Wolpert D M, Goodbody S J, Husain M, 1998 "Maintaining internal representations: the role of the human superior parietal lobe" Nature Neuroscience 1 529-533

Wilke M, Logothetis N K, Leopold D A, 2003 "Generalized flash suppression of salient visual targets" Neuron 39 1043-1052

Wilson H R, Blake R, Lee S H 2001 “Dynamics of travelling waves in visual perception” Nature 412 907910 


\section{Figure Captions}

Figure 1. The four types of bistable stimuli used in Experiment 1 are illustrated. a. Structure-from-Motion (SFM). Dots can be perceived as a rotating cylinder and the perceived direction of rotation alternates spontaneously. b. Binocular Rivalry (BR). Two square gratings (orthogonal to each other) are viewed dichoptically. Percept alternates between the two gratings (eyes). c. Necker Cube (NC). One surface of the cube appear in front and then the same surface is perceived in rear. d. Apparent Motion (AM). Two frames are alternately shown. In one frame, two disks are at the upper-left and lower-right positions, and in the other frame, they are at the upper-right and lower-left positions. The correspondence between these disks across the two frames is ambiguous. Therefore, this stimulus is typically perceived as two disks moving along either horizontal or vertical axis, and these two percepts alternates.

Figure 2. The results of Experiment 1. a. The frequency of perceptual reversals is shown with respect to flash (time zero). The frequency is shown in a normalized scale, that is, as the probability that a reversal occurs within a certain bin $(200 \mathrm{~ms})$ after a flash. For comparison, dashed lines are drawn to indicate the frequency of reversals without the flash obtained in the control experiment. The histograms for the four types of bistable stimuli show that observers' responses to reversals frequently occurred at $0.5-1.0$ seconds after a flash. This pattern was evident in all four stimuli. The data are from four observers. b. Alternation rate for each condition. The rate was higher in the condition with flash (white bars) than in the control condition (gray bars).

Figure 3. The results of Experiment 2. The frequency of perceptual reversals is shown with respect to flash (time zero). The frequency is shown in a normalized scale, that is, as the probability that a reversal occurs within a certain bin $(250 \mathrm{~ms})$ after a flash. a. The results for the condition where SFM and flash were presented in the same visual field (LVF). Alternations occurred frequently just after the flash $(<1000 \mathrm{~ms})$. b. The results

for the condition where SFM and flash were presented on the different visual field. The 
peak after the flash is not as evident as the ipsilateral condition. c. The result of NCipsilateral condition. $\mathbf{d}$. The result of NC-contralateral condition.

Figure 4. The results of Experiment 3. a. The stimulus used in condition 1 is schematically illustrated. Two SFM cylinders, rotating around the vertical axis, are presented in the LVF 0.6 deg off the horizontal meridian. The observers reported the reversals of the top cylinder (target). b. The stimulus used in the condition 2. The configuration is the same as the condition 1, but the target was the bottom cylinder. $\mathbf{c}$ and d. The stimuli used in conditions 4-5, respectively. The non-target cylinder was rotating around the horizontal axis. e-h. The alternation frequency of the condition shown in a-d in which the flash was presented at the target cylinder. The histogram shows the alternation frequency as a function of the time elapsed after a flash. The bin width is 0.5 s. The histogram is normalized by the total number of flashes. i-l. The results of the same conditions (a-d) in which the flash was presented at the non-target cylinder (bottom).

Figure 5. The results of Experiment 3 (condition 3). a. The occurrences of anti-rotation are shown as a function of the time elapsed after a flash. The histogram is normalized by the total number of flashes. $\mathbf{b}$. The durations of anti-rotation are shown. The histogram is normalized by the total number of the occurrences of anti-rotation.

Figure 6. The design and the results of the adaptation experiment (Experiment 3). a. The stimuli used in Experiment 3 are illustrated. Ambiguous figures (NC and SFM) were presented next to the fixation cross throughout a trial. After a variable duration of adaptation, a flash was presented in the background. This was followed by $500 \mathrm{~ms}$ of observation. b. The results of NC condition. Proportion of trials in which observers perceived an alternation is plotted as a function of the adaptation duration. c. The results of SFM condition. Proportion of trials in which observers perceived an alternation is plotted as a function of the adaptation duration.

Figure 7. A schematic model of the flash-induced alternation. Two mutually exclusive percepts are illustrated as the two energy wells (left and right). a. Upon stimulus onset, 
energy wells for both percepts are deep. b. When a flash is presented without a substantial adaptation, the smoothing effect (see text) is not sufficient to cause the energy wall low enough for perceptual alternation to occur. c. After adaptation, the energy well for the dominant percept becomes shallow (here the left well). d. When a flash is presented after adaptation, the smoothing effect of the flash causes the energy wall sufficiently low for the perceptual state to transit to the other interpretation.

Figure 8. a) bi-stability model: two opponent neurons inhibit each other. b) histogram of the intervals between perceptual reversals for experimental data (Experiment 2, SFMcontra) vs. simulation. c) Simulated effect of the flash. Flash onset was $5 \mathrm{sec}$ after onset of each simulated trial. d) Flash by itself does not result in any effect in the model. Flash occurs at the onset of simulated trial, thus the neurons are not adapted at the time of the flash. Figures are based on 500 simulated trials with a time-step of $10 \mathrm{~ms}$. 
Figure 1. Kanai et al.

a

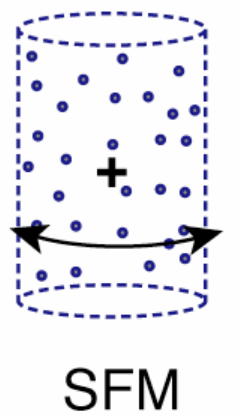

C

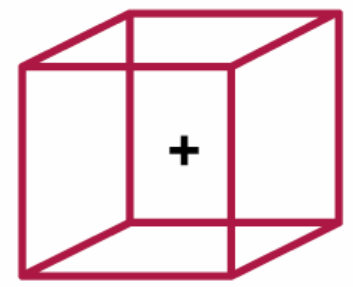

NC b
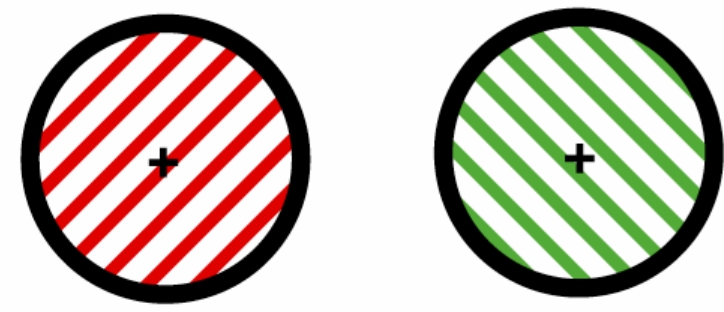

BR

d

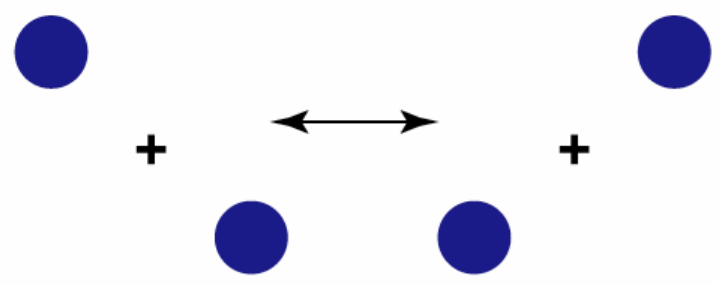

AM 
Figure 2. Kanai et al.

a
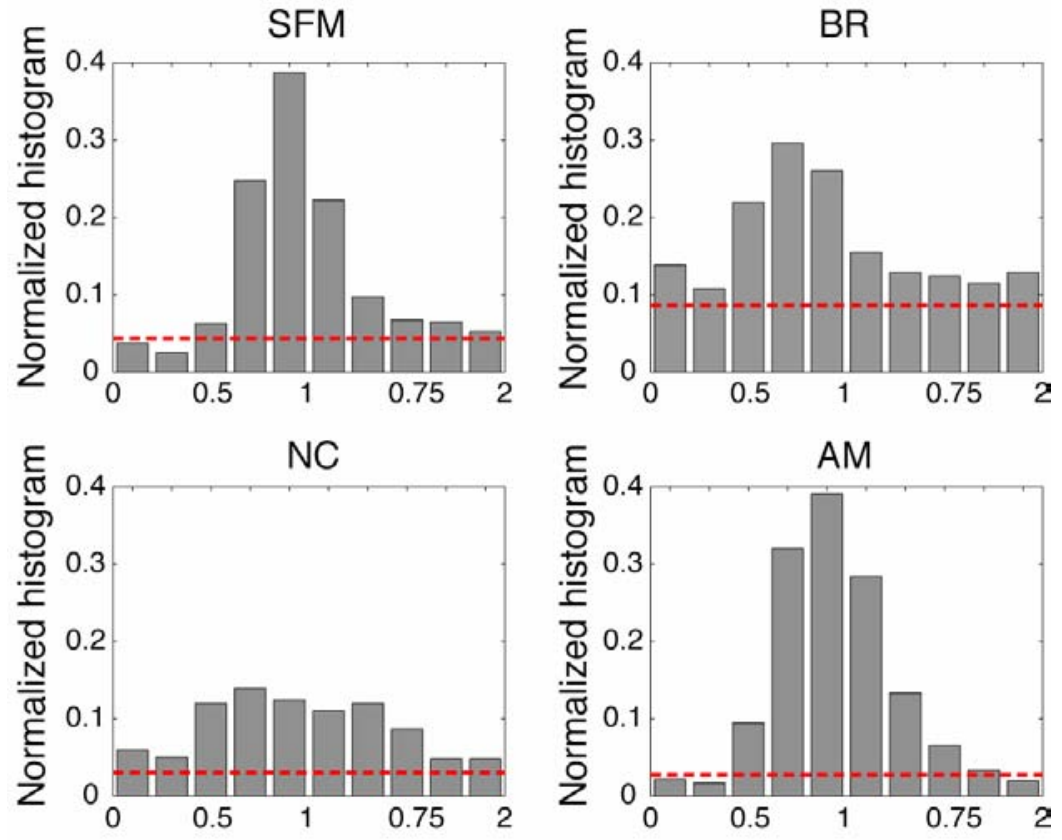

b
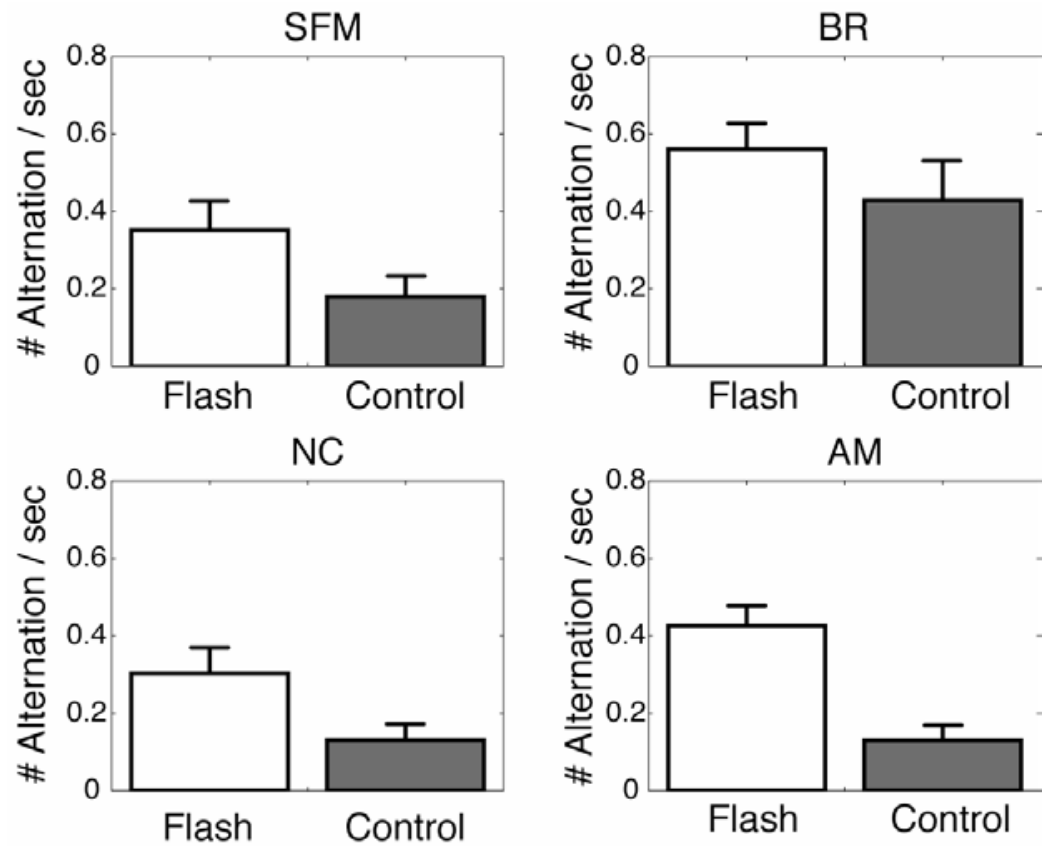
Figure 3. Kanai et al.

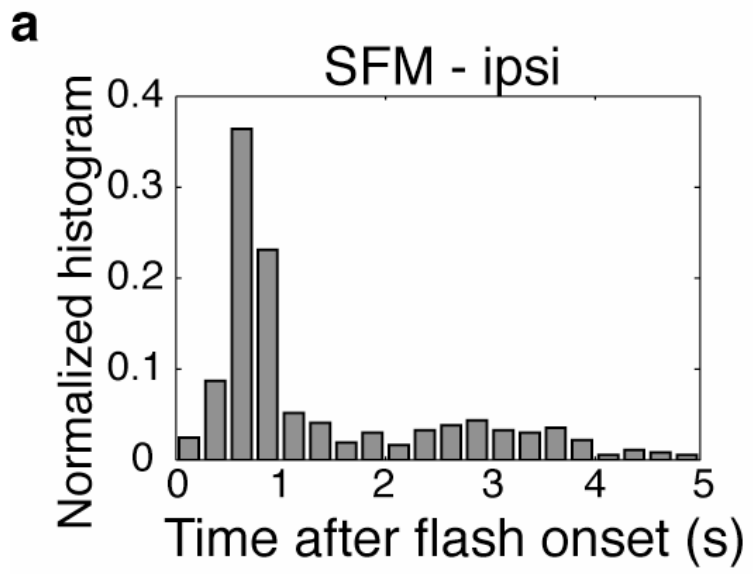

b
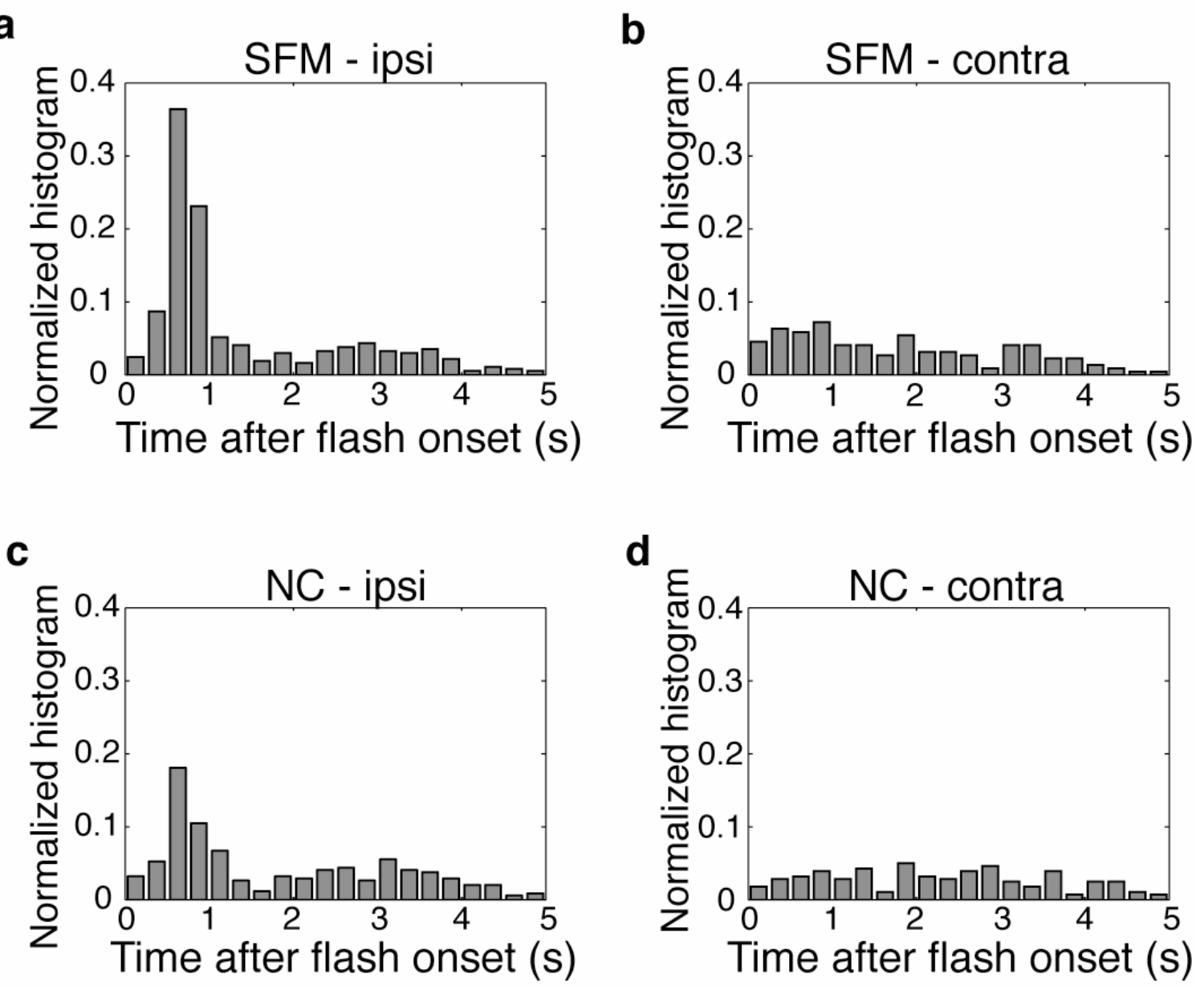
Figure 4. Kanai et al.

a
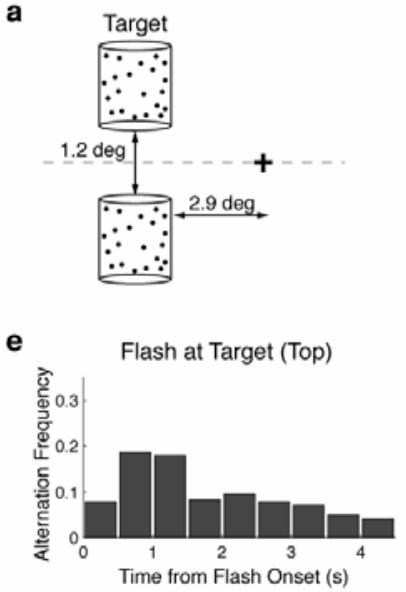

i

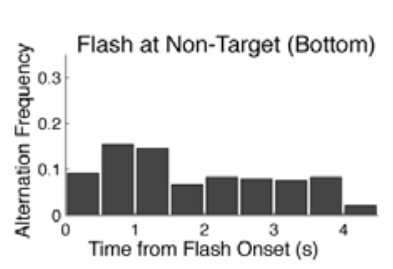

b
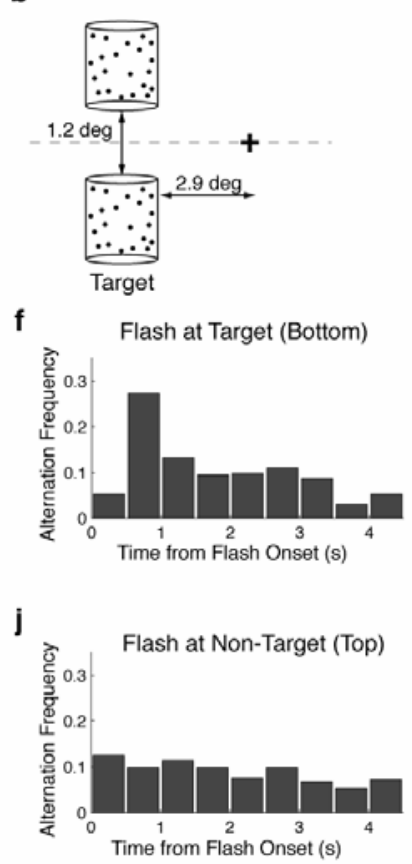

Coaxial Conditions c
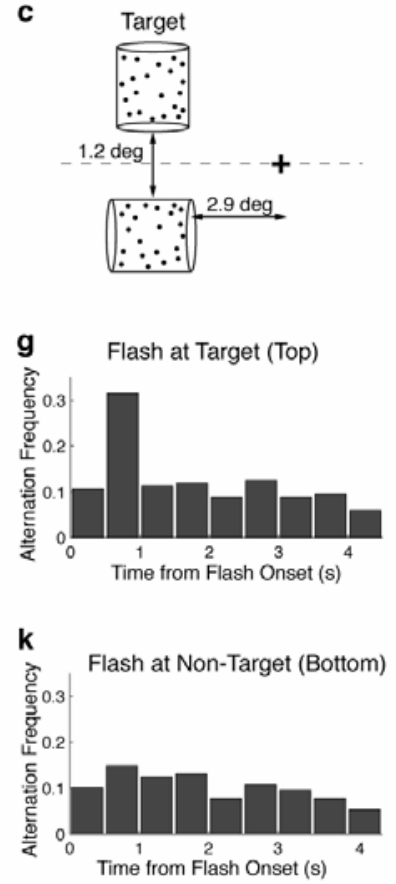

Orthogonal Conditions d
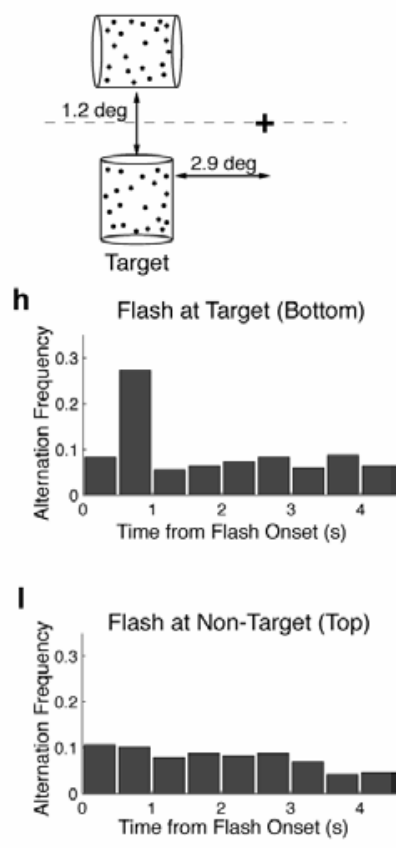
Figure 5. Kanai et al.
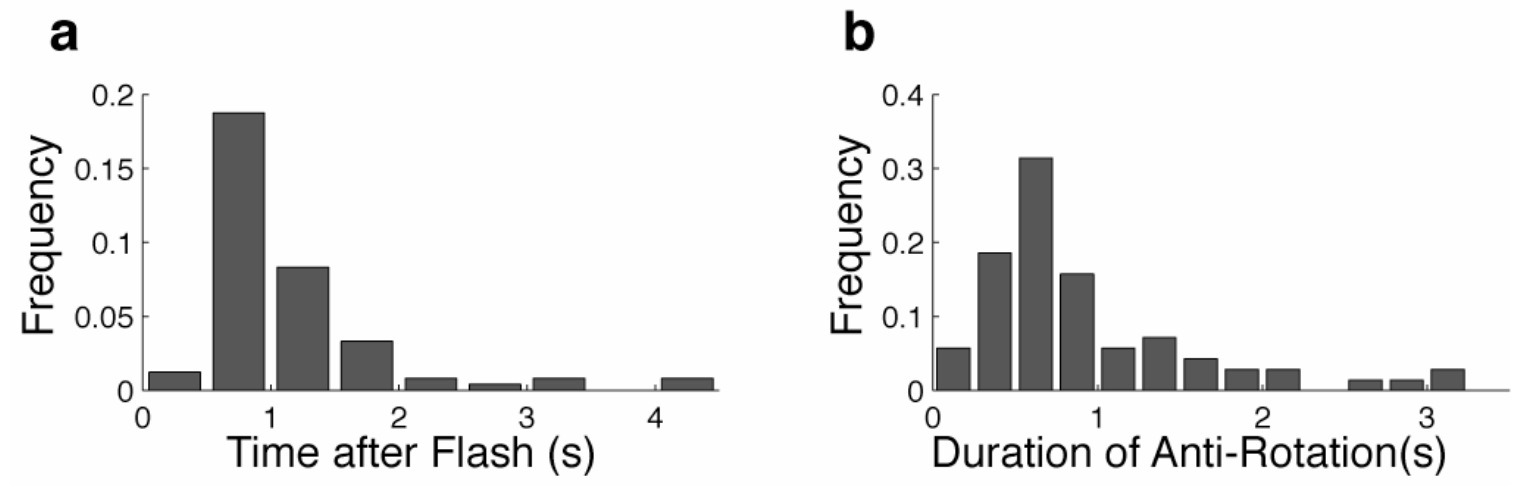
Figure 6. Kanai et al.

a
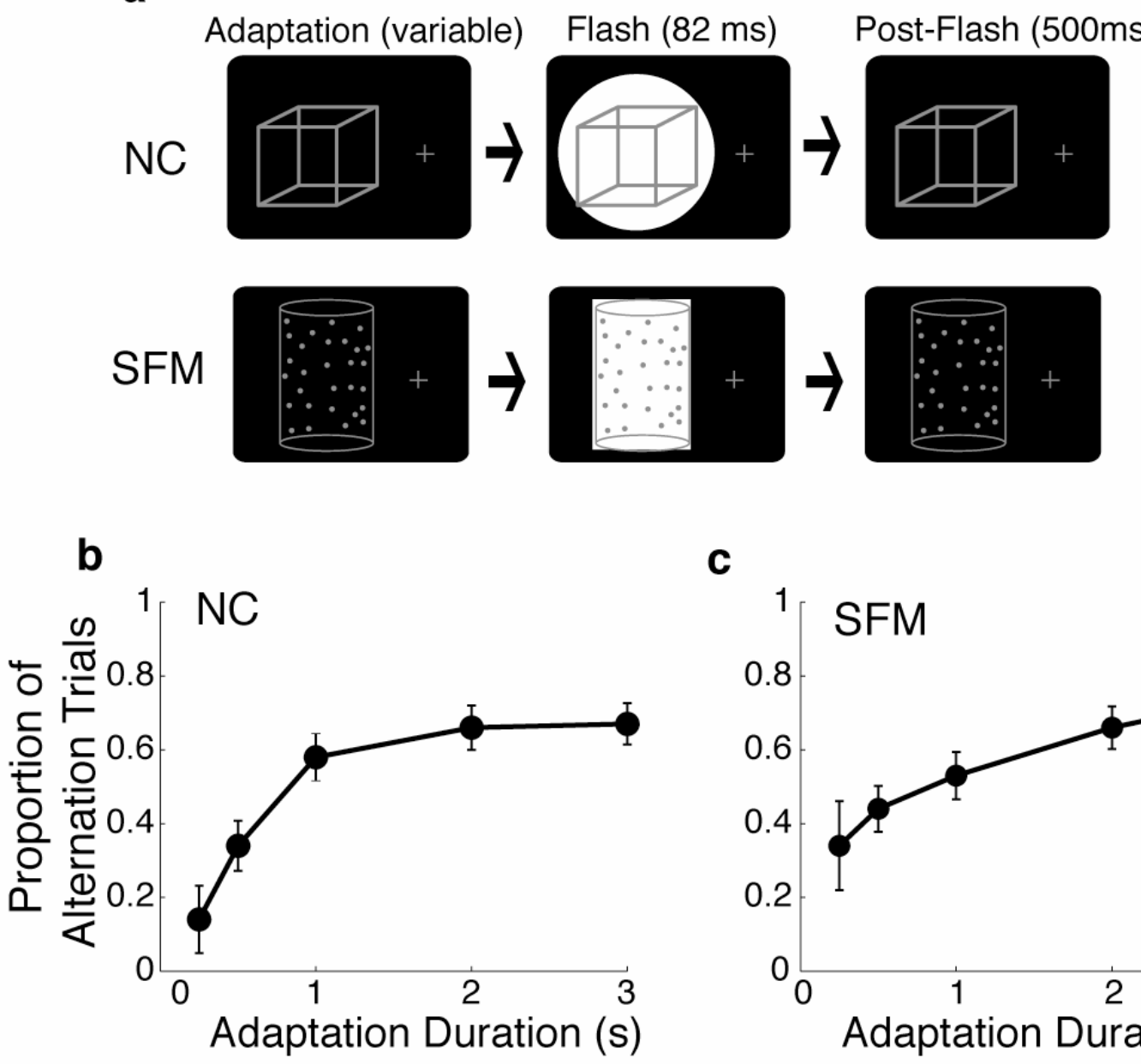

C

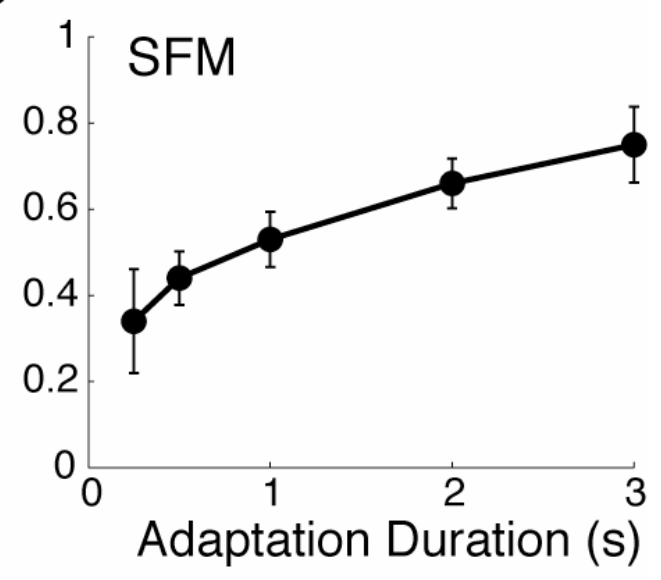


Figure 7. Kanai et al.

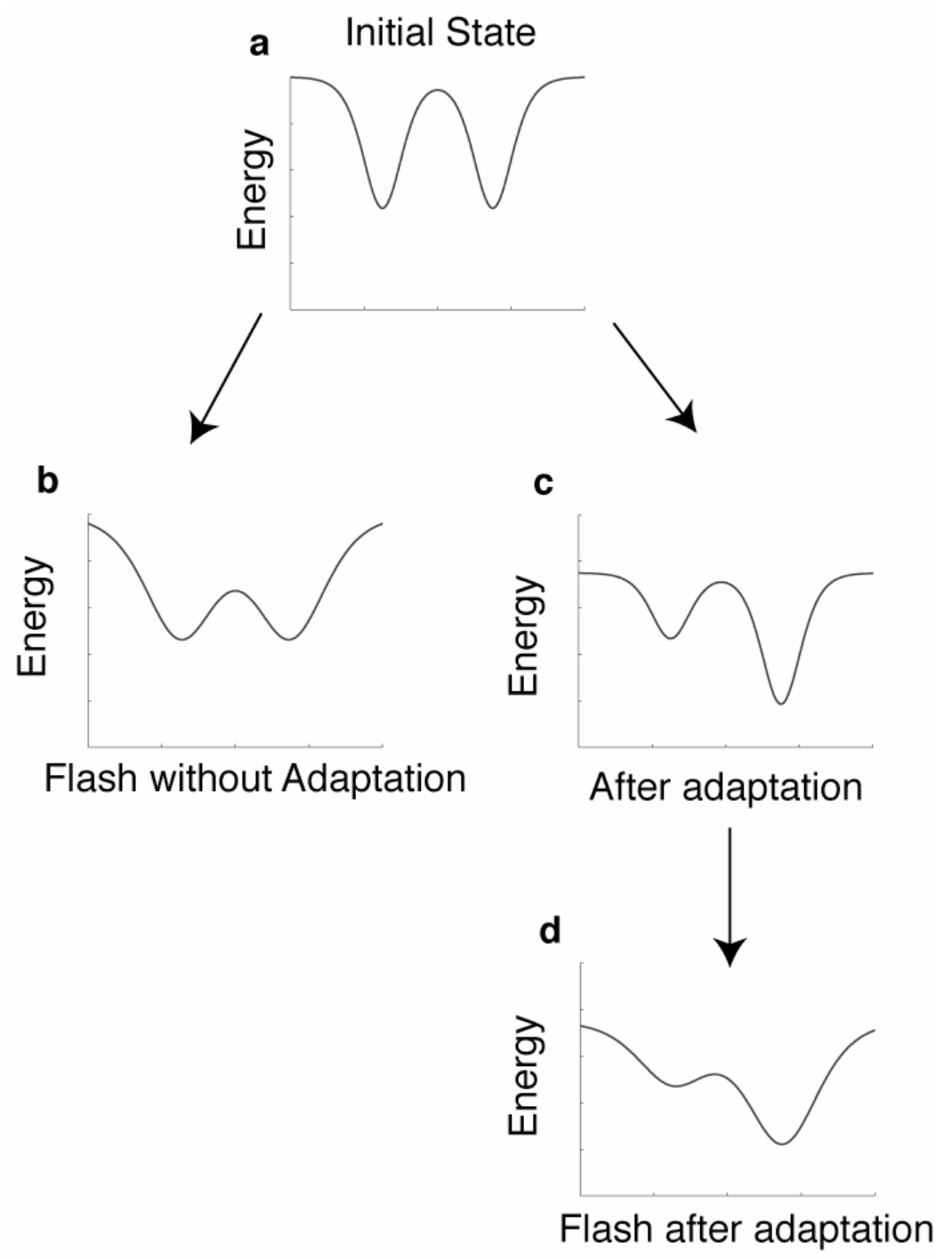


Figure 8. Kanai et al.

a

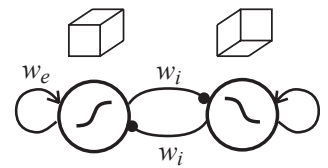

b

Emperical inter-reversal interval histogram (SFM-contra)

Simulated inter-reversal interval histogram
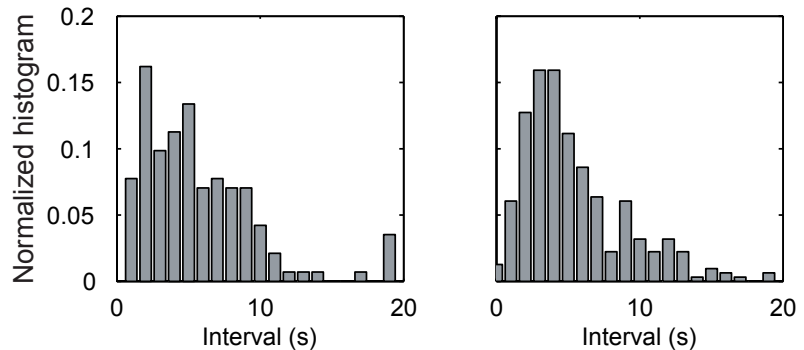

C

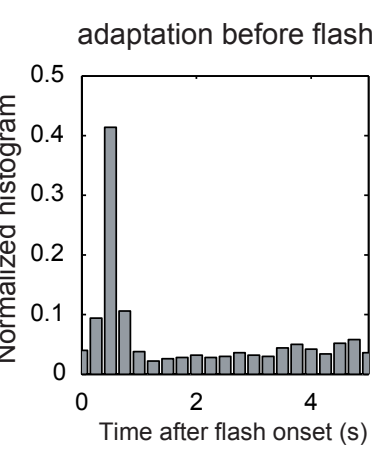

d

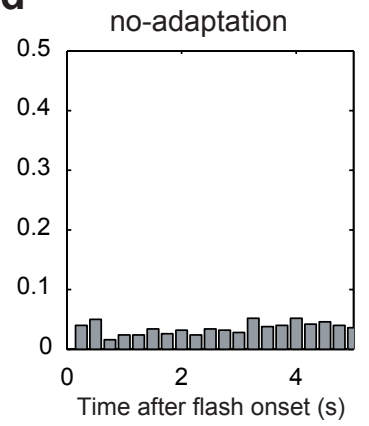




\section{Table 1}

\begin{tabular}{|c|c|c|c|}
\hline Condition & $\begin{array}{c}\text { Freq reversals at target } \\
\text { location }\end{array}$ & $\begin{array}{c}\text { Freq reversals at non- } \\
\text { target location }\end{array}$ & \\
\hline \multicolumn{4}{|l|}{ Coaxial Cylinders } \\
\hline $\begin{array}{l}\text { Target at the Top } \\
\text { (conditions 1a) }\end{array}$ & $18.8 \pm 2.5 \%$ & $15.4 \pm 2.3 \%$ & $p=0.4$ \\
\hline $\begin{array}{l}\text { Target at the Bottom } \\
\quad \text { (condition 2a) }\end{array}$ & $27.3 \pm 2.7 \%$ & $9.8 \pm 1.8 \%$ & $* * *$ \\
\hline \multicolumn{4}{|l|}{ Orthogonal Cylinders } \\
\hline $\begin{array}{l}\text { Target at the Top } \\
\text { (conditions } 1 \mathrm{~b} \text { ) }\end{array}$ & $31.5 \pm 3.6 \%$ & $14.9 \pm 2.7 \%$ & $* * *$ \\
\hline $\begin{array}{l}\text { Target at the Bottom } \\
\quad \text { (condition } 2 \mathrm{~b})\end{array}$ & $27.3 \pm 3.0 \%$ & $10.2 \pm 2.1 \%$ & $* * *$ \\
\hline
\end{tabular}

